\title{
Femtosecond Laser Induced Nanostructures in Soda-lime Glass
}

\author{
Md. Shamim Ahsan ${ }^{* 1, * 2}$ and Man Seop Lee ${ }^{* 1}$ \\ ${ }^{*}$ Photonics Application Lab, Korea Advanced Institute of Science and Technology (KAIST), 335 \\ Gwahak-ro, Yuseong-gu, Daejeon 305-701, South Korea \\ ${ }^{* 2}$ Electronics and Communication Engineering Discipline, School of Science, Engineering and \\ Technology, Khulna University, Khulna-9208, Bangladesh \\ E-mail:shamim@kaist.ac.kr
}

\begin{abstract}
We demonstrate the formation of nanostructures inside the microholes on soda-lime glass surface by irradiation with femtosecond laser pulses. Self-organized periodic nanogratings, oriented perpendicular to the laser polarization, are observed at the bottom of the microholes. The period of the nanogratings are ranging from $170 \mathrm{~nm}$ to $200 \mathrm{~nm}$. We also report the formation of nano-ripples on the side walls of the microholes, the period of which are independent of the laser fluence and the number of laser pulses. Nano-cones, with a diameter varied from $100 \mathrm{~nm}$ to $250 \mathrm{~nm}$, are also discovered on the sidewalls of the microholes. Randomly distributed spherical-shaped nanostructures with a diameter ranging from $10 \mathrm{~nm}$ to $60 \mathrm{~nm}$ are also evident inside the microholes, especially, on the top of the nano-ripples and nano-cones. We also describe, briefly, the dependence of various micro/nano-structures on the laser fluence and the number of applied laser pulses in each spot. Finally, we explain the formation mechanism of all of the micro/nano-scale features, produced during our experiment.

DOI:10.2961/jlmn.2012.02.0013
\end{abstract}

Keywords: Femtosecond Laser, nano-grating, nano-ripple, nano-cone, micro/nano-structure

\section{Introduction}

In recent years, femtosecond lasers have been considered as a versatile tool in micro/nano-machining of various materials including glasses [1-3], metals [4-6], polymers $[7,8]$, and semiconductors $[9,10]$, due to its advantages over other available technologies such as chemical, semiconductor, and ion-beam processing. Femtosecond lasers have the ability of precise micro/nanopatterning of various materials without any melting zone, micro cracks, recast layer, and shock wave, which advantages make the emergence of femtosecond lasers to the forefront of the research field.

Femtosecond lasers have been widely used for the last few decades in micro/nano-machining of soda-lime glass due to its diverse application fields, especially, in manufacturing optical data storage and integrated optical devices $[11,12]$. For these kinds of applications, the precise generation of micro/nano-holes is one of the key structures among all kinds of laser induced patterns.

In some recent studies, the researchers have reported the fabrication of micro/nano-holes on the surface of and inside soda-lime glass using Single-Beam Direct Laser Writing (SBDLW) technique $[1,13,14]$. However, discovering the formation of nanostructures inside the laser induced microholes on soda-lime glass is still limited. The information regarding the interactions of femtosecond laser with soda-lime glass is still insufficient. Furthermore, the influence of laser fluence and the number of laser pulses on the size and shape of the micro/nano-structures are still inadequate. Thus, it is significant to examine the effects of the laser fluence and the number of laser pulses on the formation of micro/nano-scale features inside the microholes on soda-lime glass surface. Besides, it is desirable to investigate the appropriate mechanism behind the formation of various nanostructures inside the microholes, formed on soda-lime glass substrate.

In this report, through careful control over various laser parameters such as the laser fluence and the number of laser pulses in each spot, we demonstrate the fabrication of microholes on soda-lime glass surface. At the bottom of the microholes, self-organized periodic nanogratings are formed with a period varied from $170 \mathrm{~nm}$ to $200 \mathrm{~nm}$. The formed self-organized nanogratings are oriented perpendicular to the laser polarization direction. The dependence of the nanogratings' period on the number of laser pulses is also evident from the experimental results.

We also discover the formation of nano-ripples and nano-cones on the side walls of the microholes, fabricated on soda-lime glass surface. The evolution of the nanoripples and the nano-cones has been discussed. Nano-cones are primarily evident near the glass surface, although they are also observed at the bottom of the microholes. The period of the nano-ripples is almost constant, which value is in the sub-wavelength range, i.e., less than the wavelength of the incident laser beam $\left(\lambda_{\mathrm{i}}\right)$. The diameter of the nano-cones is diverse, which is ranging from $100 \mathrm{~nm}$ to $250 \mathrm{~nm}$. The nano-ripples and the nano-cones are observed significantly at low laser fluences. These kinds of nanostructures start to disappear with the increase of the laser fluence. We also report the formation of randomlyoriented spherical-shaped nanostructures on the top of the nano-ripples and the nano-cones, the diameter of which is varied from $10 \mathrm{~nm}$ to $60 \mathrm{~nm}$. Unlike nano-ripples and nano-cones, randomly distributed nanostructures are evident at any laser fluence and number of laser pulses irradiated in each spot. We also describe, briefly, the 
dependence of elliptical shaped microholes' dimensions on the laser fluence and the number of irradiated laser pulses. Finally, we thoroughly illustrate the effects of the laser fluence and the number of laser pulses on the formation of various nano-scale features, produced inside the microholes, and their controlling process.

\section{Experimental Details}

We carried out our experiment using a Ti:sapphire femtosecond laser, the central wavelength of which was $786.5 \mathrm{~nm}$. The pulse width and the pulse repetition rate of the ultra-short laser pulses were $183 \mathrm{fs}$ and $1 \mathrm{kHz}$. Sodalime glass sample, with a thickness of $1 \mathrm{~mm}$ and a refractive index $(\eta)$ of $\sim 1.51$ at $786.5 \mathrm{~nm}$, were used in the experiment.

We focused the laser beam vertically on the top of the sample surface through a $20 \times$ achromatic objective lens, which has a numerical aperture of NA $=0.42$. The schematic diagram of the experimental setup is shown in Fig. 1.

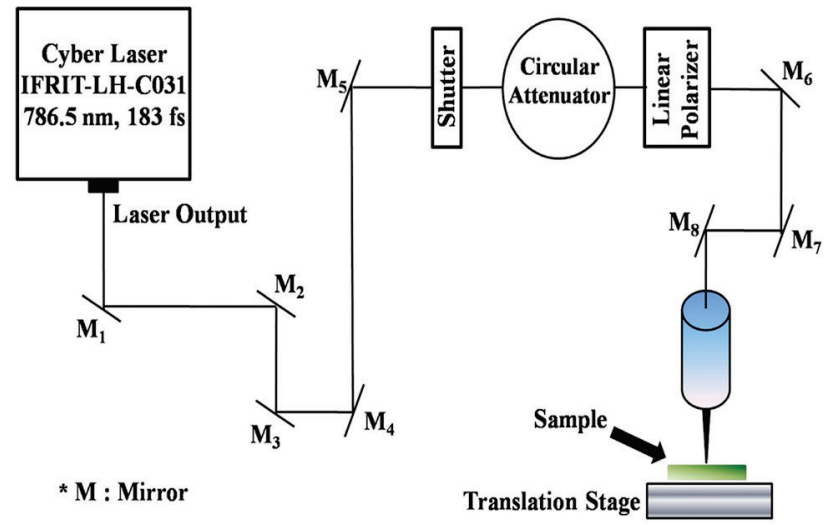

Fig. 1 Schematic diagram of the experimental setup.

The soda-lime glass sample was mounted on a 3-axis linear translation stage, which has a resolution of $100 \mathrm{~nm}$ in the $\mathrm{x}, \mathrm{y}$, and $\mathrm{z}$ directions. In order to control the exposure time, i.e., the number of laser pulses in each spot on the sample surface, a computer controlled high speed shutter was used. We placed a circular attenuator after the mechanical shutter to control the power of the laser pulses. A linear polarizer was placed after the circular attenuator to produce s-polarized laser beam. We also used a power meter to measure the average power of the laser pulses after the objective lens. Reflective mirrors were used to guide the laser beam onto the sample surface.

To fabricate microholes on soda-lime glass surface, we used SBDLW technique. Microholes were manufactured by varying the laser fluence from $2.14 \mathrm{~J} / \mathrm{cm}^{2}$ to $142.03 \mathrm{~J} / \mathrm{cm}^{2}$ (pulse energy was varied from $1 \mu \mathrm{J}$ to $113 \mu \mathrm{J}$ ). The number of laser pulses in each spot was ranging from 500 to 3500 . In order to analyze the micro/nano-scale features, we investigated the images of the microholes under a Scanning Electron Microscope (SEM). Before taking the SEM images, the sample was cleaned in an ultrasonic bath with de-ionized water at room temperature. Due to the nonconducive property of soda-lime glass, the sample was sputter coated with a thin $(\sim 30 \mathrm{~nm})$ layer of gold to provide conductivity for SEM examination.

\section{Results}

We produced microholes on soda-lime glass surface by controlling various irradiation conditions of a single beam femtosecond laser such as the laser fluence and the number of applied laser pulses in each spot. A variety of nano-scale structures, such as self-organized periodic nanogratings, nano-ripples, and nano-cones were formed inside the microholes. On the top of the nano-ripples and nano-cones, randomly distributed spherical-shaped nanostructures were also evident. In this section, we briefly describe the formation of various micro/nano-scale features on sodalime glass surface.

\subsection{Microholes fabrication}

In our experiment, by controlling the laser fluence and the number of laser pulses of a femtosecond laser beam, we fabricated microholes on the sample surface. Fig. 2 shows the SEM images of the formed microholes for a wide-range of laser fluence (varied from $2.04 \mathrm{~J} / \mathrm{cm}^{2}$ to $142.03 \mathrm{~J} / \mathrm{cm}^{2}$ ) and number of laser pulses in each spot (varied from 500 to $3500)$.

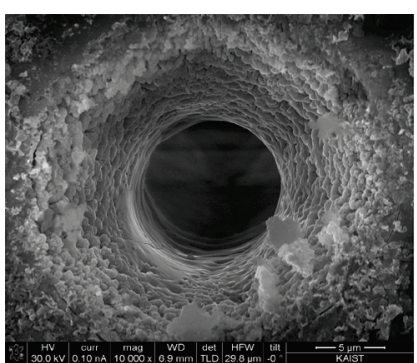

(a)

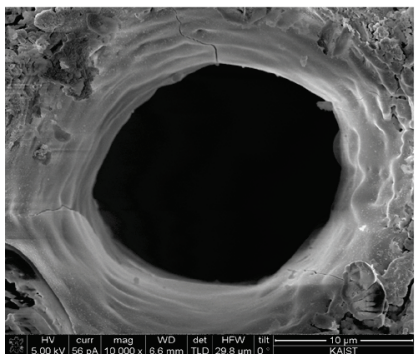

(c)

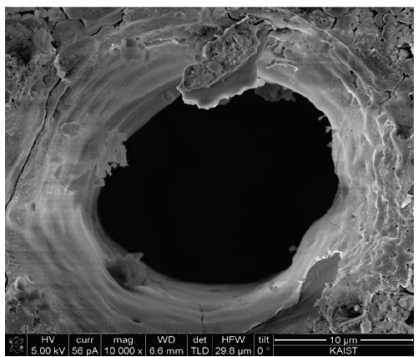

(e)

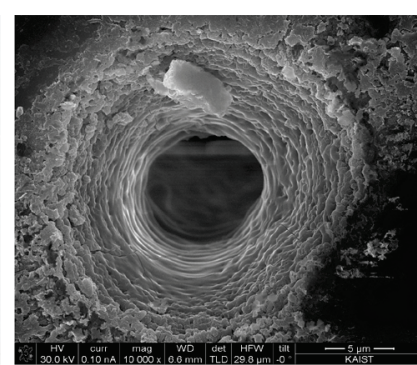

(b)

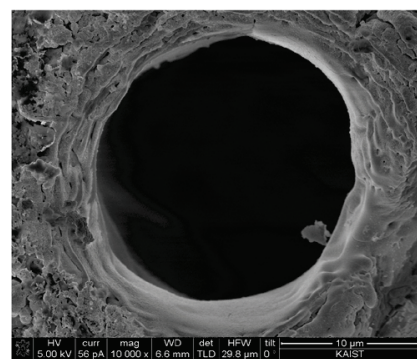

(d)

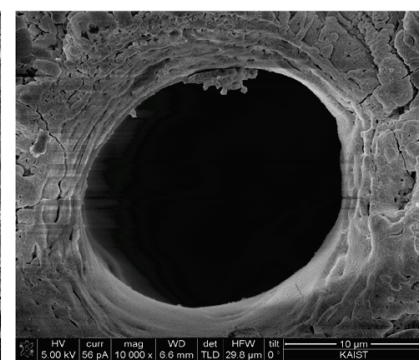

(f)
Fig. 2 SEM images of the fabricated microholes for a widerange of laser fluence $(F)$ and number of irradiated laser pulses $(N)$ in each spot. (a) $N=500, F=2.14 \mathrm{~J} / \mathrm{cm}^{2}$; (b) $N$ $=3500, F=2.14 \mathrm{~J} / \mathrm{cm}^{2}$; (c) $N=500, F=87.34 \mathrm{~J} / \mathrm{cm}^{2}$; (d) $N=3500, F=87.34 \mathrm{~J} / \mathrm{cm}^{2}$; (e) $N=500, F=142.03$ $\mathrm{J} / \mathrm{cm}^{2}$; (f) $N=3500, F=142.03 \mathrm{~J} / \mathrm{cm}^{2}$.

The fabricated microholes were elliptical in shape due to the elliptical shape of the femtosecond laser beam. From 
the experimental results, it is apparent that the dimensions and the depth of the microholes increased with the increase of the laser fluence.

With the increase of the number of laser pulses in each spot, the dimensions of the microholes also increased. The change in the size of the microholes due to the change in the number of laser pulses is less significant compared to the change in the size of the microholes due to the change in the laser fluence, which was varying in the range of 2.14 $\mathrm{J} / \mathrm{cm}^{2}$ to $142.03 \mathrm{~J} / \mathrm{cm}^{2}$. Fig. 3 shows the relationship between the length of the microholes' axes (major and minor) with the laser fluence and the number of irradiated laser pulses in each spot.

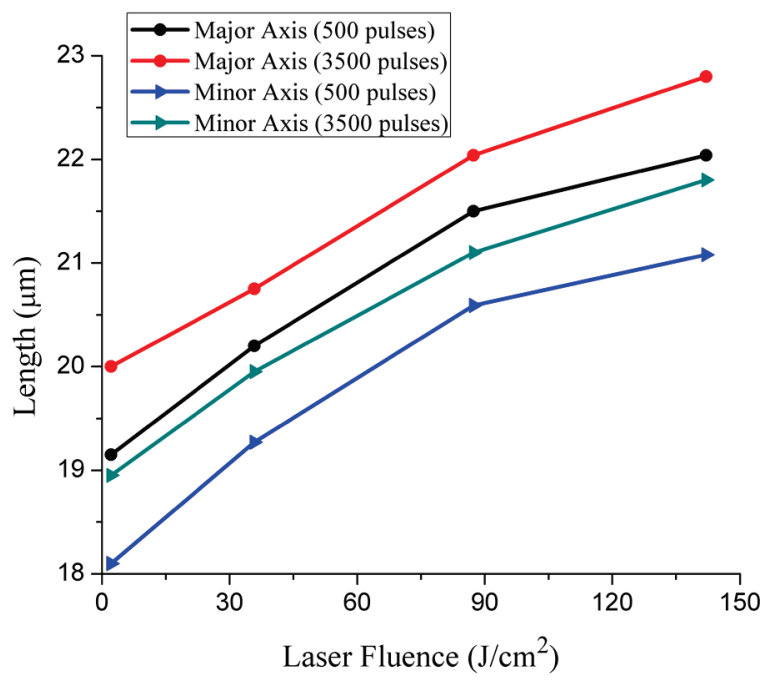

Fig. 3 Dependence of the length of the microholes' axes on the laser fluence and the number of laser pulses in each spot.

\subsection{Formation of self-organized periodic nanogratings}

Self-organized periodic nanogratings were formed at the bottom of the microholes, which is evident from the SEM images of Fig. 4. The nanogratings were perpendicularly oriented to the laser polarization direction as proposed in [1].

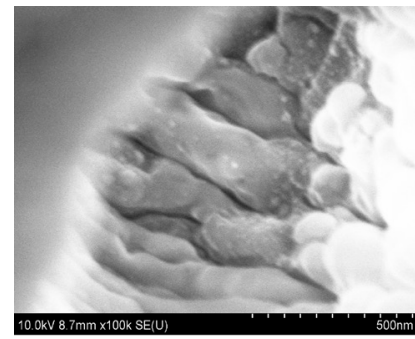

(a)

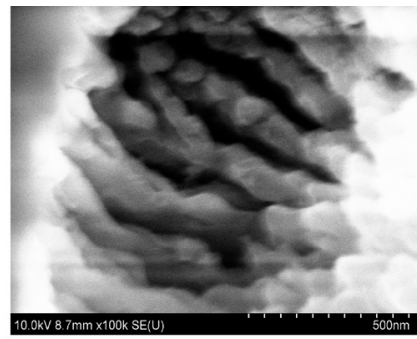

(b)
Fig. 4 SEM images of the self-organized periodic nanogratings, produced at the bottom of the microholes in soda-lime glass at laser fluence of $2.14 \mathrm{~J} / \mathrm{cm}^{2}$. (a) After 1000 pulses; (b) after 3500 pulses.

The period of the self-organized periodic nanogratings were ranging from $170 \mathrm{~nm}$ to $200 \mathrm{~nm}$, which period was dependent on the number of laser pulses irradiated in each spot. Due to the increase of the laser pulses, the period of the self-organized nanogratings were decreased. Unlike nanogratings' period, the depth of the nanogratings increased with the increase of the number of laser pulses in each spot. Since the depth of the microholes was increased with increasing pulse energy, it was impossible to take the SEM images at the bottom of the microholes for high laser energy. As a result, more experiments are required to investigate the influence of laser energy on the nanogratings' period.

\subsection{Formation of nanostructures inside the microholes}

Various kinds of nanostructures, such as nano-ripples, nano-cones, and randomly distributed nanostructures, were evident inside the microholes, fabricated on the sample surface. On the side walls of the microholes, nano-ripples with constant period were formed, the value of which is in the sub-wavelength range as shown in Fig. 5.

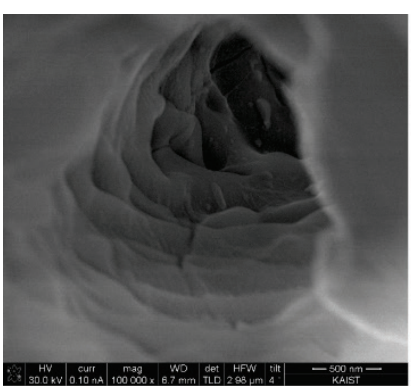

(a)

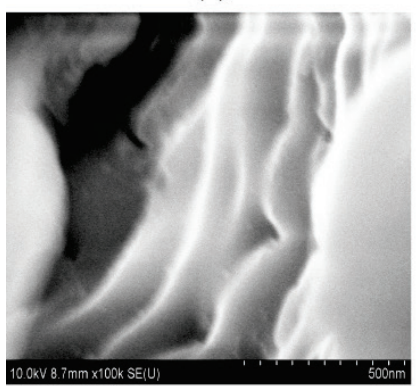

(c)

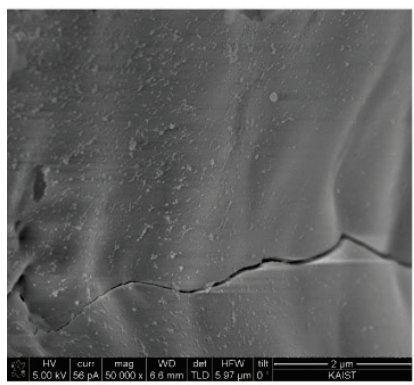

(e)

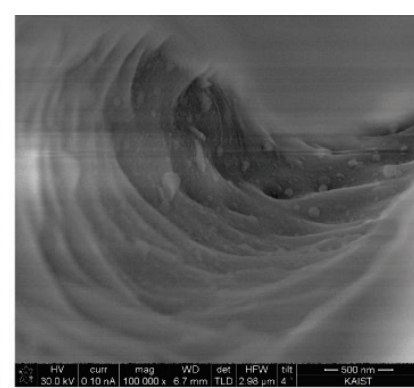

(b)

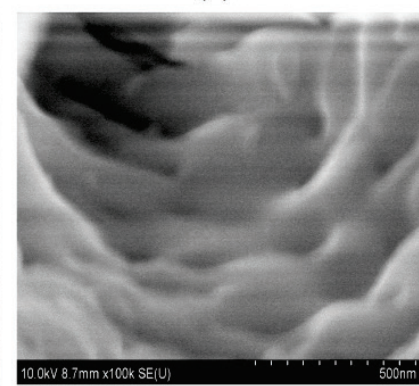

(d)

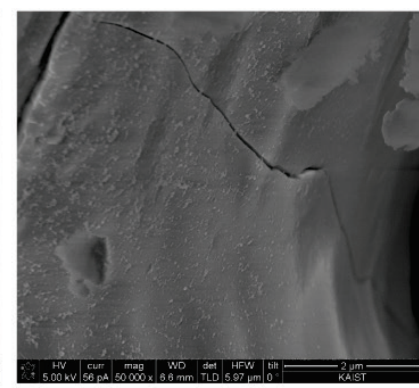

(f)
Fig. 5 SEM images of the nano-ripples formed on the side walls of the microholes, produced on soda-lime glass surface. (a) After 500 laser pulses $(N)$ at laser fluence $(F)$ of $2.14 \mathrm{~J} / \mathrm{cm}^{2}$; (b) $N=2500, F=2.14 \mathrm{~J} / \mathrm{cm}^{2}$; (c) $N=1500, F=$ $19.1 \mathrm{~J} / \mathrm{cm}^{2}$; (d) $N=3500, F=19.1 \mathrm{~J} / \mathrm{cm}^{2}$; (e) $N=500, F=$ $87.34 \mathrm{~J} / \mathrm{cm}^{2}$; (f) $N=500, F=142 \mathrm{~J} / \mathrm{cm}^{2}$.

The appearance of nano-ripples, on the side walls of the microholes, was certainly dependent on the laser fluence and the number of applied laser pulses. Nano-ripples were observed significantly all over the side walls, inside the 
microholes, typically at low laser fluences. This kind of nano-metric structures almost disappeared at high laser fluence. On the contrary, when small number of laser pulses was applied at low laser fluence, nano-ripples were formed extensively on the side walls of the microholes. With the increase of the laser pulses, nano-ripples started to depart from the side walls of the microholes as shown in Figs $5(a-d)$. At high laser fluence, especially at $113 \mu \mathrm{J}$, we didn't observe any nano-ripples even for small number of laser pulses as shown in Figs. 5(e and f). Micro-cracks were also evident in some places on the side walls of the microholes, when high laser fluence was used as depicted in Figs. 5(e and f).

In parallel with the nano-ripples, nano-cones were also evident on the side walls of the fabricated microholes, near the sample surface as illustrated in Figs. 6(a, c, d, and e).

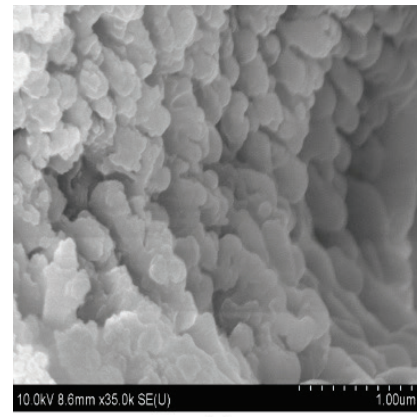

(a)

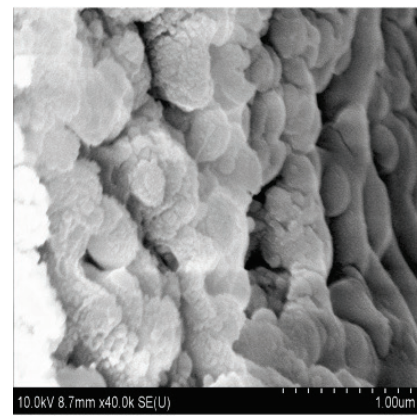

(c)

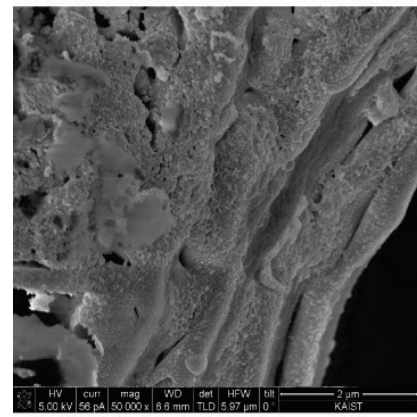

(e)

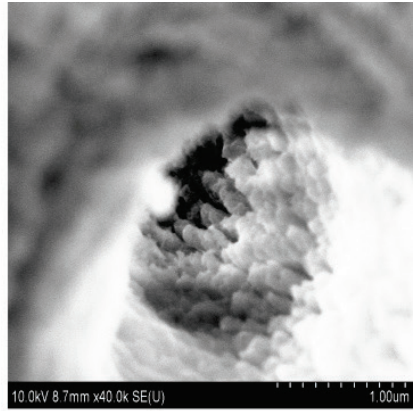

(b)

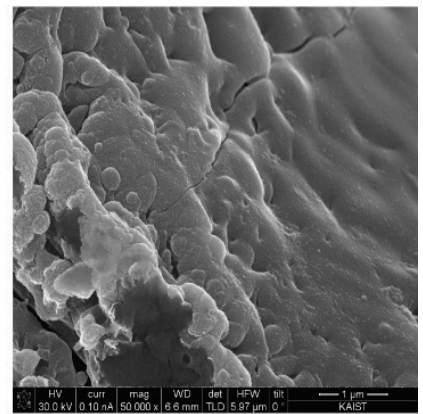

(d)

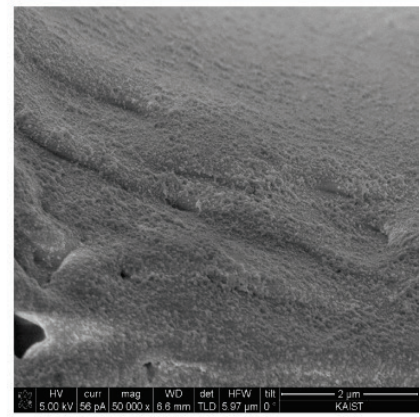

(f)
Fig. 6 SEM images of the nano-cones formed on the side walls of the microholes, produced on soda-lime glass surface. (a) After 500 laser pulses $(N)$ at laser fluence $(F)$ of $2.14 \mathrm{~J} / \mathrm{cm}^{2}$; (b) $N=2000, F=2.14 \mathrm{~J} / \mathrm{cm}^{2}$; (c) $N=1500, F=$ $19.1 \mathrm{~J} / \mathrm{cm}^{2}$; (d) $N=3500, F=35.79 \mathrm{~J} / \mathrm{cm}^{2}$; (e) $N=3500$, $F=87.34 \mathrm{~J} / \mathrm{cm}^{2}$; (f) $N=3500, F=142.03 \mathrm{~J} / \mathrm{cm}^{2}$.

Nano-cones were also formed near the bottom of the microholes as shown in Fig. 6(b). The diameter of the nano-cones was varied, which value was ranging from 100 $\mathrm{nm}$ to $250 \mathrm{~nm}$. We investigated that the formation of nanocones were certainly influenced by the laser fluence. At low laser fluence, nano-cones were formed considerably on the side walls of the microholes. Like nano-ripples, nano-cones showed decreasing trend in relation with the increasing laser fluence. At high laser fluence, especially, at $113 \mu \mathrm{J}$, nano-cones were disappeared absolutely from the side walls as shown in Fig. 6(f). From the experimental results, it is obvious that there is no definite impact of the number of laser pulses on the formation of nano-cones.

On the top of the nano-ripples and nano-cones, randomly oriented spherical-shaped nanostructures were formed as shown in Figs. 5 and 6. The diameter of the random nanostructures was varied from $10 \mathrm{~nm}$ to $60 \mathrm{~nm}$. Unlike nano-ripples and nano-cones, random nanostructures were observed at any laser fluence, even at $113 \mu \mathrm{J}$, as shown in Figs. 5(e and f) and 6(f).

\section{Discussion}

In this report, we exhibit the dependence of microholes' diameter on the laser fluence and the number of laser pulses irradiated in each spot as shown in Fig. 3. From the experimental results, it is evident that the size of the microholes' increases with the increase of the laser fluence. This relationship is in considerable agreement with the Gaussian beam model proposed by Joglekar et. al. [15]. Fig. 7 shows the intensity profile of the femtosecond laser beam, which certainly indicates that the laser beam was Gaussian in nature. With the increase of the laser energy, the spot size of the laser beam also increased, which in turn increase the diameter of the microholes. Furthermore, from Fig. 2 it is also apparent that the fabricated microholes are elliptical in shape. Since the shape of the femtosecond laser beam is elliptical (after the exit port, the width of the beam in the vertical direction is $4.9 \mathrm{~mm}$ and in the horizontal direction is $4.3 \mathrm{~mm}$ ), it is reasonable that the fabricated microholes' shape is elliptical.

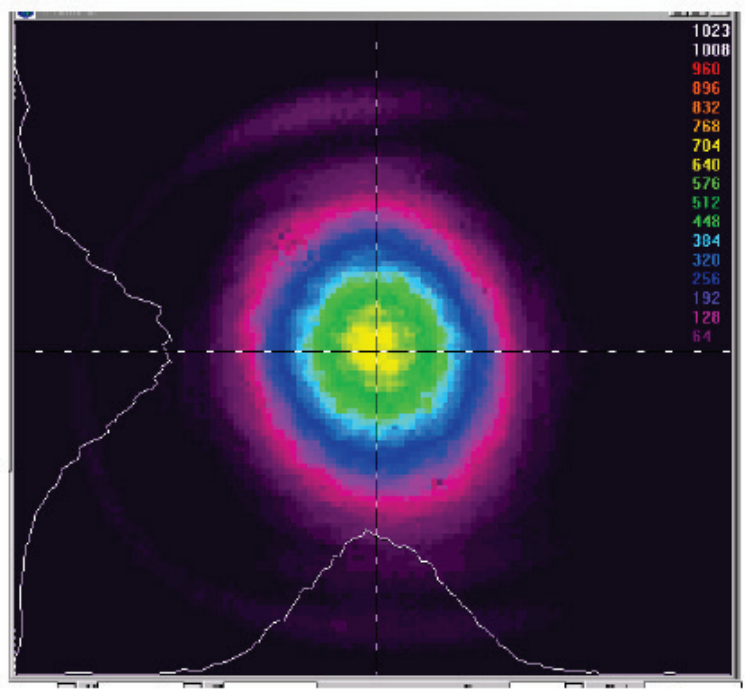

Fig. 7. Intensity profile of the laser beam after the exit port of the IFRIT.

The formation mechanism of the self-organized periodic nanogratings of Fig. 4 can be interpreted as the interaction of the high intensity femtosecond laser beam 
and the bulk plasma. As a result, periodic nanogratings are printed at the bottom of the microholes. There is a strong debate among several models in explaining the nanogratings' period. According to Bonch-Bruevich et. al. model [16], the period of the self-organized nanogratings, formed by linearly polarized laser pulses, can be obtained by the following equation.

$$
d=\frac{\lambda}{\eta \pm \sin \theta} \text { with } \mathbf{g} \perp \mathbf{E}
$$

where $d$ is the nanogratings' period, $\lambda$ is the wavelength of the incident laser beam, $\eta$ is the real part of the refractive index of the material, $\theta$ is the incident angle of the laser beam on the sample surface, $\mathbf{g}$ is the grating vector, and $\mathbf{E}$ is the electric field vector of the incident wave. Using this model, the grating period should be either $313 \mathrm{~nm}$ or 1542 $\mathrm{nm}$, which values are enormously different from our experimental values (varying from $170 \mathrm{~nm}$ to $200 \mathrm{~nm}$ ).

Shimotsuma et. al., on the other hand, demonstrate the nanogratings' period as a function of the laser fluence, where the period of the nanogratings increases with the laser fluence, which relationship can be represented as follows [17]:

$$
d=\frac{2 \pi}{\sqrt{\frac{1}{T_{e}}\left(\frac{m_{e} \omega^{2}}{3 k_{B}}-\frac{e^{2} N_{e}}{3 \varepsilon_{0} k_{B}}\right)-k_{p h}^{2}}}
$$

where $d$ is the grating period, $T_{\mathrm{e}}$ is the electron temperature, $m_{\mathrm{e}}$ is the electron mass, $\omega$ is the angular frequency, $k_{\mathrm{B}}$ is the Boltzmann's constant, $e$ is the electron charge, $N_{\mathrm{e}}$ is the electron density, $\varepsilon_{0}$ is the dielectric constant of air, $\mathrm{k}_{\mathrm{ph}}=$ $\omega \eta / c$ is the wave vector, $\eta$ is the refractive index of material, and $c$ is the speed of light. However, this model is unable to explain the dependence of nanogratings' period on the number of laser pulses properly. Besides, the model becomes invalid at a high plasma density (although lower than critical) by predicting a complex period, which invalidates the model under breakdown conditions.

One of the most remarkable model, proposed by Bhardwaj et. al. [18] for the nanogratings inside the bulk of a glass represents that the nanogratings' period is constant and independent of the laser fluence and the number of laser pulses. This model is further refined by Buividas et. al. [19], where the researchers showed that the model proposed by Bhardwaj et. al. is also suitable for surface ripples (i.e. self-formed nanogratings) at the dielectric breakdown conditions when ultra-short laser pulses are applied. According to these models, the grating period for our case should be equal to $\lambda_{\mathrm{i}} / 2 \eta=786.5 /(2 \times 1.51)=260$ $\mathrm{nm}$, which is quite different than our experimental results. Thus, none of the above models can explain our experimental results completely.

The variation of the nanogratings period with the number of irradiated laser pulses can be interpreted in the following manner. As we know, the plasma frequency $\omega_{P}$ is a function of the real part of the refractive index, which is defined by the equation as follows [20]:

$$
\omega_{P}^{2}=\frac{\eta e^{2}}{\varepsilon_{0} m}
$$

where $e$ is the electron's charge, $\varepsilon_{0}$ is the dielectric constant of vacuum, and $m$ is the effective optical mass of electron.
Since, the self-organized nanogratings are formed by applying a large number of laser pulses at the same spot, the dielectric constant of the glass is changed during the experiment due to the variation of the relaxation time of electron $(\tau)$ and $\omega_{P}$. The electron temperature $T_{e}$, as well as $\tau$ and $\omega_{P}$ are variable depending on the number of overlapped laser pulses. The $\tau$ decreases with $T_{e}$ caused by the heating of the electron subsystem $\left(\tau \propto 1 / T_{e}^{2}\right)$, which also increases $\omega_{P}$ [21]. These phenomena might have caused the variation of the refractive index with the number of overlapped laser pulses, which influence the variation of nanogratings' period with the number of irradiated laser pulses.

The nano-ripples and nano-cones are formed due to the variation of the electric field of the femtosecond laser beam. After the initial laser pulses, the structure of the soda-lime glass surface is altered due to the influence of the external electric field. The modified internal structure of the sample glass in turn causes the alteration of the external electric field [22]. From Fig. 2, it is significant that the surface of the side walls of the microholes is curved and, as a consequence, laser polarization is different at different position inside the microholes. The interaction of the external electric field of the laser beam and the bulk plasma waves causes a periodic intensity variation, which consequently gives rise to the production of periodic nanoripples on the side walls of the microholes. Besides, the Gaussian beam might have influenced the production of periodic nano-ripples as well. Due to the Gaussian pattern of the laser beam, the depth of the microholes at the center increases faster than the surroundings [23]. This phenomenon might have caused the production of periodic nano-ripples. However, due to the increase of the number of laser pulses in each spot leads to the break of the nanoripples and consequently nano-cones have appeared on the side walls of the microholes. It is already proven by researchers that the period of the nano-ripples is wavelength $\left(\lambda_{\mathrm{i}}\right)$ dependent, which can be obtained by the equation as follows [24]:

$$
d_{1}=\lambda_{i} /\left(\eta^{2}-\sin ^{2} \theta\right)^{1 / 2} \text { with } \mathbf{r} \perp \mathbf{E}
$$

where $d_{1}$ is the period of the nano-ripples, $\theta$ is the incident angle of the laser beam on the microholes' wall, $\mathbf{r}$ is the nano-ripple vector, and $\mathbf{E}$ is the electrical field vector of the incident beam.

Furthermore, the randomly distributed spherical-shaped nanostructures are formed on the top of the nano-ripples and nano-cones all over the microholes. Unlike nanoripples and nano-cones, random nanostructures are independent of the laser fluence and the number of laser pulses. These nanostructures might be the re-deposited particles during laser machining.

\section{Conclusion}

In summary, we demonstrated the development of variety of nano-scale features inside the microholes, where the microholes are fabricated on soda-lime glass surface by controlling the irradiation conditions of a femtosecond laser beam. The major and minor axes of the elliptical shaped microholes were increasing with the increase of the laser fluence and the number of laser pulses in each spot. 
Self-organized nanogratings, with a period ranging from $170 \mathrm{~nm}$ to $200 \mathrm{~nm}$, were evident at the bottom of the microholes. The self-formed nanogratings were formed vertically to the laser polarization direction. Nano-ripples and nano-cones were also formed on the side walls of the microholes. Nano-ripples' period was in the subwavelength range, the value of which was almost constant. Unlike nano-ripples, nano-cones' diameter was variable, which was varying in the range of $100 \mathrm{~nm}$ to $250 \mathrm{~nm}$. We also reported the formation of randomly distributed spherical-shaped nanostructures all-over the microholes, especially, on the top of the nano-ripples and the nanocones. The diameter of the spherical-shaped nanostructures was varied diversely, which values were ranging from 10 $\mathrm{nm}$ to $60 \mathrm{~nm}$. We also explained, briefly, the dependence of various micro/nano-metric structures on the laser fluence and the number of applied laser pulses. Above all, we thoroughly explained the formation mechanism of all of the micro/nano-structures. We strongly believe that, our research will play important roles in understanding the interactions of femtosecond laser pulses with soda-lime glass and characterizing various laser induced micro/nanometric structures.

\section{Acknowledgements}

This work was supported by the Brain Korea 21 Project, the School of Information Technology, KAIST in 2012.

\section{References}

[1] M. S. Ahsan, Y. G. Kim and M. S. Lee: J. Non Cryst. Solids, 357, (2011) 851.

[2] Q. Zhang, H. Lin, B. Jia, L. Xu and M. Gu: Opt. Express, 18, (2010) 6885.

[3] F. Ahmed, M. S. Lee, H. Sekita, T. Sumiyoshi and M. Kamata: Appl. Phys. A, 93, (2008) 189.

[4] L. Qi, K. Nishii and Y. Namba: Opt. Lett., 34, (2009) 1864.

[5] A. Y. Vorobyev and C. Guo: Opt. Express, 14, (2006), 2164.
[6] M. S. Ahsan, Y. G. Kim and M. S. Lee: Proc. SPIE, 7925, (2011) p.792512.

[7] M. Alubaidy, K. Venkatakrishnan and B. Tan: J. Micromech. Microeng., 20, (2010), 055012.

[8] J. Si, Z. Meng, S. Kanehira, J. Qiu, B. Hua and K. Hirao: Chem. Phys. Lett., 399, (2004) 276.

[9] L. Ji-Ming and X. Jian-Ting: Laser Phys., 18, (2008) 1539.

[10]T. Sakai, N. Nedyalkov and M. Obara: J. Phys. D, 40, (2007) 7485

[11] Y. Li, W. Watanabe, K. Itoh and Z. Sun: Appl. Phys. Lett., 81, (2002) 1952.

[12]K. Minoshima, A. M. Kowalevicz, E. P. Ippen and J. G. Fujimoto: Opt. Express, 10, (2002) 645.

[13] Y. Zhou, M. H. Hong, J. Y. H. Fuh, L. Lu, B. S. Lukyanchuk and Z. B. Wang: J. Alloys. Compd., 449, (2008), 246.

[14]A. Zoubir, L. Shah, K. Richardson and M. Richardson: Appl. Phys. A, 77, (2003) 311.

[15]A. P. Joglekar, H. Liu, G. J. Spooner, E. Meyhöfer, G. Mourou and A. J. Hunt: Appl. Phys. B, 77, (2003) 25.

[16]A. M. Bonch-Bruevich, M. N. Libenson, V. S. Makin, and V. V. Trubaev: Opt. Engg., 31, (1992) 718.

[17] Y. Shimotsuma, P. G. Kazansky, J. Qiu and K. Hirao: Phys. Rev. Lett., 91, (2003) 247405.

[18] V. R. Bhardwaj, E. Simova, P. P. Rajeev, C. Hnatovsky, R. S. Taylor, D. M. Rayner and P. B. Corkum: Phys. Rev. Lett., 96, (2006) 057404.

[19]R. Buividas, L. Rosa, R. Šliupas, T. Kudrius, G. Šlekys, V. Datsyuk, S. Juodkazis: Nanotechnology, 22, (2011) 055304.

[20]S. A. Maier: "Plasmonics: Fundamentals and Applications" (Springer, USA, 2007) p. 10.

[21]Z. Lin, L. V. Zhigilei: Phys. Rev. B, 77 (2008) 075133.

[22]P. A. Temple and M. J. Soileau: IEEE J. Quantum Electron, 17, (1981) 2067.

[23] M. Zhou, H. F. Yang, B. J. Li, J. Dai, J. K. Di, E. L. Zhao and L. Cai: Appl. Phys. A, 94, (2009) 571.

[24] Y. Huang, S. Liu, W. Li, Y. Liu and W. Yang: Opt. Express, 17, (2009) 20756.

(Received: May 11, 2011, Accepted: April 16, 2012) 